\title{
A note on regularity for free convolutions
}

\author{
Serban Teodor Belinschi ${ }^{\mathrm{a}, \mathrm{b}, 1}$ \\ a Mathematics Department, Indiana University, Bloomington, IN 47405, USA \\ ${ }^{\mathrm{b}}$ Institute of Mathematics of the Romanian Academy, P.O. Box 1-764, RO-014700, Bucharest, Romania
}

Received 5 January 2004; received in revised form 8 April 2005; accepted 18 May 2005

Available online 7 December 2005

\begin{abstract}
Let $\mu \boxplus v$ and $\mu \otimes v$ denote the free additive convolution and the free multiplicative convolution, respectively, of the Borel probability measures $\mu$ and $\nu$. We analyze the boundary behavior of the functions $G_{\mu \boxplus v}(z)=\int \frac{1}{z-t} \mathrm{~d}(\mu \boxplus \nu)(t)$ and $\psi_{\mu \otimes \nu}(z)=$ $\int \frac{z t}{1-z t} \mathrm{~d}(\mu \otimes v)(t)$. We prove that, under certain conditions, these functions extend continuously to the boundary of their natural domains as functions with values in the extended complex plane $\mathbb{C} \cup\{\infty\}$. As a consequence, we obtain that $\mu \boxplus v$ (respectively $\mu \otimes v)$ can never be purely singular, unless $\mu$ or $v$ is concentrated in one point.
\end{abstract}

Crown Copyright $\odot 2005$ Published by Elsevier SAS. All rights reserved.

\section{Résumé}

Soit $\mu \boxplus v$ et $\mu \otimes v$ la convolution additive libre et, respectivement, la convolution multiplicative libre des mesures boréliennes de probabilité $\mu$ et $\nu$. Nous étudions le comportement à la frontière des fonctions $G_{\mu \boxplus v}(z)=\int \frac{1}{z-t} \mathrm{~d}(\mu \boxplus v)(t)$ et $\psi_{\mu \boxplus v}(z)=$ $\int \frac{z t}{1-z t} \mathrm{~d}(\mu \otimes v)(t)$. Nous démontrons que, dans certaines conditions, ces fonctions peuvent être prolongées à la frontière de leurs domaines naturels de définition, comme fonctions continues prenant des valeurs dans la compactification $\mathbb{C} \cup\{\infty\}$ du plan complexe $\mathbb{C}$. Une conséquence de ce fait est que $\mu \boxplus v$ (et, respectivement, $\mu \otimes v$ ) peut être purement singulière seulement si $\mu$ ou $v$ est concentrée en un point.

Crown Copyright $\odot 2005$ Published by Elsevier SAS. All rights reserved.

MSC: primary 46L54; secondary 30D40

Keywords: Free convolutions; Cluster sets of analytic functions

\section{Introduction}

Free convolutions appear as natural analogues of the classical convolutions in the context of free probability theory. Denote by $\mathcal{M}$ the set of Borel probability measures supported on the real line $\mathbb{R}$, by $\mathcal{M}_{+}$the ones supported on the interval $[0,+\infty)$, and by $\mathcal{M}_{*}$ the ones supported on the unit circle in the complex plane $\mathbb{C}$ having nonzero first moment.

E-mail addresses: sbelinsc@math.uwaterloo.ca, teodor.belinschi@imar.ro (S.T. Belinschi).

1 Present address: Department of Pure Mathematics, University of Waterloo, Waterloo, Ontario N2L 3G1, Canada. 
For $\mu, \nu \in \mathcal{M}$ one defines the free additive convolution $\mu \boxplus \nu$ of $\mu$ and $\nu$ in the following way: $\mu \boxplus v$ is the probability distribution of $X+Y$, where $X$ and $Y$ are free selfadjoint random variables with distribution $\mu$ and $\nu$.

Similarly, given $\mu, v \in \mathcal{M}_{*}$, the free multiplicative convolution $\mu \otimes v$ of $\mu$ and $v$ is defined as the probability distribution of $X Y$, where $X$ and $Y$ are free unitary random variables with distribution $\mu$ and $\nu$.

For $\mu, v \in \mathcal{M}_{+}$, one still can define $\mu \nabla v$, but in a slightly different way: $\mu \otimes v$ is the distribution of $X^{1 / 2} Y X^{1 / 2}$, where $X$ and $Y$ are free positive random variables with distributions $\mu$ and $\nu$. (We refer to [16] for an introduction to the area of free probability theory.)

Let $\mathbb{C}^{+}=\{z \in \mathbb{C}: \Im z>0\}$ denote the upper half of the complex plane, $\mathbb{D}=\{z \in \mathbb{C}:|z|<1\}$ the open unit disk, and let $\mathbb{T}=\{z \in \mathbb{C}:|z|=1\}$ denote the unit circle in the complex plane.

For any $\mu \in \mathcal{M}$, let

$$
G_{\mu}(z)=\int_{-\infty}^{\infty} \frac{1}{z-t} \mathrm{~d} \mu(t), \quad \Im z \neq 0,
$$

denote the Cauchy transform of $\mu$, and let $F_{\mu}(z)=\frac{1}{G_{\mu}(z)}$. By results of [5] (see Corollary 5.8), there exists a number $M \geqslant 0$, depending on $\mu$, such that $F_{\mu}$ has a right inverse $F_{\mu}^{-1}$ defined on $\{x+\mathrm{i} y: y>M,|x|<y\}$; the function $\phi_{\mu}(z)=F_{\mu}^{-1}(z)-z$ has the remarkable property that

$$
\phi_{\mu}(z)+\phi_{\nu}(z)=\phi_{\mu \boxplus v}(z)
$$

for $z \in\{x+\mathrm{i} y: y>M,|x|<y\}$, with $M$ large enough.

Similar results have been proved for free multiplicative convolutions. For $\mu \in \mathcal{M}_{+}$(or $\mu \in \mathcal{M}_{*}$ ), let

$$
\psi_{\mu}(z)=\int_{V} \frac{z t}{1-z t} \mathrm{~d} \mu(t),
$$

where $z$ belongs either to the open unit disk $\mathbb{D}$ (if $\mu \in \mathcal{M}_{*}$, in which case $V=\mathbb{T}$ ), or to $\mathbb{C} \backslash\left[0, \infty\right.$ ) (if $\mu \in \mathcal{M}_{+}$, in which case $V=[0,+\infty)$ ). The function $\psi_{\mu}$ determines uniquely the measure $\mu$, and it is univalent in a neighborhood of zero (if $\mu \in \mathcal{M}_{*}$ ) or in the left half-plane $\mathbb{i}^{+}=\{z \in \mathbb{C}: \Re z<0\}$ (if $\mu \in \mathcal{M}_{+}$).

Let $\eta_{\mu}(z)=\psi_{\mu}(z) /\left(1+\psi_{\mu}(z)\right)$. The function $\Sigma_{\mu}(z)=\frac{1}{z} \eta_{\mu}^{-1}(z)$ satisfies the equation

$$
\Sigma_{\mu 凶 v}(z)=\Sigma_{\mu}(z) \Sigma_{v}(z)
$$

for $z$ in a neighborhood of zero (if $\mu, v \in \mathcal{M}_{*}$ ), or in a neighborhood of the interval $(-\varepsilon, 0)$ for some $\varepsilon>0$ (if $\mu, v \in \mathcal{M}_{+}$). For proofs we refer to [5] and [14].

Previous results related to regularity questions for free convolutions of probability measures seem to indicate that these operations typically do not favor the existence of a large singular part (with respect to the Lebesgue measure). It has been proved in [6] and, respectively, in [1], that after free additive, respectively multiplicative, convolution, atoms usually disappear. More precisely, $c$ is an atom for $\mu \boxplus v$ if and only if there exist atoms $a$ of $\mu$ and $b$ of $v$ with the property that $a+b=c$ and $\mu(\{a\})+v(\{b\})>1$. Moreover, $(\mu \boxplus v)(\{c\})=\mu(\{a\})+v(\{b\})-1$. A similar result holds for free multiplicative convolution. The singular continuous part has been shown to disappear altogether for the free additive convolution of a measure with itself. Also, in this case, the density of the absolutely continuous part is continuous, and analytic outside a closed set of measure zero in $\mathbb{R}$ (see [2], Theorem 3.4). Similar results for free multiplicative convolutions can be found in [3]. Biane has shown in [7] that if $\mu=\frac{1}{2 \pi t} \sqrt{4 t-x^{2}} \mathrm{~d} x$ is the semicircular distribution, then $\mu \boxplus v$ is absolutely continuous with respect to the Lebesgue measure, its density is a continuous function, analytic wherever strictly positive. These facts contrast sharply with the behavior of classical convolution.

In this paper we show that, roughly speaking, the singular continuous part of the free convolution of two measures, none of them a point mass, if existing, has its (topological) support included in the support of the absolutely continuous part. Moreover, the Lebesgue measure of the singular continuous support is always zero. It is easy to see that these results have no classical counterpart. Indeed, consider a probability measure $v$ which is purely singular continuous and has its topological support equal to the interval $[0,1]$. Its classical convolution with $\left(\delta_{-4}+\delta_{4}\right) / 2$ will give a purely singular measure.

An important tool in proving results related to regularity for free convolutions has been subordination of analytic functions. The following result has been first proved in [15] under some more restrictive hypotheses, and later in [8] in full generality (see Theorems 3.1, 3.5, and 3.6): 


\section{Theorem 1.1.}

(a) Let $\mu, v \in \mathcal{M}$ be two Borel probability measures on the real line. Then there is an analytic function $\omega_{\mu, v}$ on $\mathbb{C} \backslash \mathbb{R}$ such that

(a1) $F_{\mu \boxplus v}(z)=F_{\mu}\left(\omega_{\mu, v}(z)\right), z \in \mathbb{C} \backslash \mathbb{R}$

(a2) $\omega_{\mu, v}(\bar{z})=\overline{\omega_{\mu, v}(z)}$, $\Im \omega_{\mu, v}(z) \geqslant \Im z$ for $z \in \mathbb{C}^{+}$, and $\lim _{y \rightarrow+\infty}\left(\omega_{\mu, v}(\mathrm{i} y) / \mathrm{i} y\right)=1$.

The map $\omega_{\mu, v}$ is uniquely determined by properties (a1) and (a2).

(b) Let $\mu, v \in \mathcal{M}_{*}$. There exists an analytic function $\omega_{\mu, v}$ defined on $\mathbb{D}$ such that

(b1) $\left|\omega_{\mu, \nu}(z)\right| \leqslant|z|, z \in \mathbb{D}$;

(b2) $\psi_{\mu}\left(\omega_{\mu, v}(z)\right)=\psi_{\mu \otimes_{v}}(z), z \in \mathbb{D}$.

The map $\omega_{\mu, v}$ is uniquely determined by properties (b1) and (b2).

(c) Let $\mu, v \in \mathcal{M}_{+}$be different from $\delta_{0}$. There exists an analytic function $\omega_{\mu, v}$ defined on $\mathbb{C} \backslash[0,+\infty)$, such that

(c1) If $z \in \mathbb{C}^{+}$, then $\omega_{\mu, v}(z) \in \mathbb{C}^{+}, \omega_{\mu, v}(\bar{z})=\overline{\omega_{\mu, v}(z)}$ and $\arg \left(\omega_{\mu, v}(z)\right) \geqslant \arg (z)$;

(c2) $\psi_{\mu}\left(\omega_{\mu, v}(z)\right)=\psi_{\mu \otimes v}(z), z \in \mathbb{C} \backslash[0,+\infty)$.

The map $\omega_{\mu, v}$ is uniquely determined by properties $(\mathrm{c} 1)$ and $(\mathrm{c} 2)$.

The results stated in the following lemma have been proved in [6] and [1], and are direct consequences of Theorem 1.1, equalities (1) and (2), and of analytic continuation.

\section{Lemma 1.2.}

(a) Let $\mu, v \in \mathcal{M}$. With the notations from Theorem 1.1, the following equality holds:

$$
F_{\mu \boxplus v}(z)+z=\omega_{\mu, v}(z)+\omega_{v, \mu}(z), \quad z \in \mathbb{C} \backslash \mathbb{R} .
$$

(b) Let $\mu, v \in \mathcal{M}_{+}\left(\right.$or $\left.\mu, v \in \mathcal{M}_{*}\right)$. With the notations from Theorem 1.1, the following equality holds:

$$
\psi_{\mu 凶 v}(z)=\frac{\omega_{\mu, v}(z) \omega_{\nu, \mu}(z)}{z-\omega_{\mu, \nu}(z) \omega_{\nu, \mu}(z)}
$$

or, equivalently,

$$
\eta_{\mu \bigotimes v}(z)=\frac{1}{z} \omega_{\mu, v}(z) \omega_{v, \mu}(z)
$$

for all $z \in \mathbb{C} \backslash[0,+\infty)($ or $z \in \mathbb{D}$, respectively).

For a function $f: \mathbb{D} \rightarrow \mathbb{C} \cup\{\infty\}$, and a point $x \in \mathbb{T}$, we say that the nontangential limit of $f$ at $x$ exists if the $\operatorname{limit}_{\lim _{z \rightarrow x}, z \in \Gamma_{\alpha}(x)} f(z)$ exists for all $\alpha \in(0, \pi)$, where $\Gamma_{\alpha}(x)$ denotes the angular domain having vertex $x$, angular opening $\pi-\alpha$, and being bisected by the radius of $\mathbb{D}$ that ends at $x$. A similar definition holds for maps defined on the upper half-plane. If $f: \mathbb{C}^{+} \rightarrow \mathbb{C} \cup\{\infty\}$ and $x \in \mathbb{R}$, then the nontangential limit of $f$ at $x$ exists if $\lim _{z \rightarrow x, z \in \Gamma_{\alpha}(x)} f(z)$ exists for all $\alpha \in(0, \pi)$, where $\Gamma_{\alpha}(x)$ denotes the angular domain having vertex $x$, angular opening $\pi-\alpha$, and being bisected by the perpendicular on $\mathbb{R}$ at $x$. We say that nontangential limit at infinity exists if $\lim _{z \rightarrow \infty, z \in \Gamma_{\alpha}(0)} f(z)$ exists, for all $\alpha \in(0, \pi)$. We shall denote nontangential limits by $\varangle \lim _{z \rightarrow \alpha} f(z)$, or

$$
\lim _{z \rightarrow \infty} f(z) .
$$

The following three theorems describe properties of analytic functions in the unit disc related to their nontangential boundary behavior.

Theorem 1.3. Let $f: \mathbb{D} \rightarrow \mathbb{C}$ be a bounded analytic function. Then the set of points $\mathrm{e}^{\mathrm{i} \theta} \in \mathbb{T}$ at which the radial limit $\lim _{r \rightarrow 1} f\left(r \mathrm{e}^{\mathrm{i} \theta}\right)$ of $f$ fails to exist is of linear measure zero.

Theorem 1.4. Let $f: \mathbb{D} \rightarrow \mathbb{C}$ be a bounded analytic function, and let $\mathrm{e}^{\mathrm{i} \theta} \in \mathbb{T}$. If there exists a path $\gamma:[0,1) \rightarrow \mathbb{D}$ such that $\lim _{t \rightarrow 1} \gamma(t)=\mathrm{e}^{\mathrm{i} \theta}$ and $\ell=\lim _{t \rightarrow 1} f(\gamma(t))$ exists in $\mathbb{C}$, then the nontangential limit of $f$ at $\mathrm{e}^{\mathrm{i} \theta}$ exists, and equals $\ell$. 
Theorem 1.5. Let $f: \mathbb{D} \rightarrow \mathbb{C}$ be an analytic function. Assume that there exists a set $A$ of nonzero linear measure in $\mathbb{T}$ such that the nontangential limit of $f$ exists at each point of $A$, and equals zero. Then $f(z)=0$ for all $z \in \mathbb{D}$.

Theorem 1.3 is due to Fatou, Theorem 1.5 to Privalov, and Theorem 1.4 to Lindelöf. For proofs, we refer to [10] (see Theorems 2.1, 2.3, and 8.1). Observe that, by Theorems 1.3 and 1.4, the existence of radial and nontangential limits are equivalent for bounded analytic maps defined in the unit disc.

Let us notice that, in particular, all the above theorems apply to self-maps of the unit disc. The upper half-plane is known to be conformally equivalent to the unit disc via a rational transformation of the extended complex plane: the map $g(z)=(z-\mathrm{i}) /(z+\mathrm{i})$ is a conformal bijection of $\mathbb{C} \cup\{\infty\}$ onto itself that carries $\mathbb{C}^{+}$onto $\mathbb{D}$ and $\mathbb{R} \cup\{\infty\}$ onto $\mathbb{T}$; a subset of $\mathbb{R}$ has Lebesgue measure zero if and only if its image via $g$ is a set of linear measure zero in $\mathbb{T}$. Moreover, such rational transformations preserve angles. Thus, even if the map $g$ does not necessarily carry an angular domain onto an angular domain, we have however that $f$ has nontangential limit at $g(x) \in \mathbb{T}$ if and only if $f \circ g$ has nontangential limit at $x \in \mathbb{R} \cup\{\infty\}$. In particular, all the above theorems apply to analytic self-maps of the upper half-plane, or to analytic maps from $\mathbb{C}^{+}$into $-\mathbb{C}^{+}$.

Main ingredients of our proofs will be two results from the theory of cluster sets of analytic functions. Given a domain (i.e. an open connected set) $D \subseteq \mathbb{C} \cup\{\infty\}$, the cluster set of a function $f: D \rightarrow \mathbb{C} \cup\{\infty\}$ at the point $P \in \bar{D}$ is

$$
C(f, P)=\left\{z \in \mathbb{C} \cup\{\infty\} \mid \exists\left\{z_{n}\right\}_{n \in \mathbb{N}} \subset D \backslash P \text { such that } \lim _{n \rightarrow \infty} z_{n}=P, \lim _{n \rightarrow \infty} f\left(z_{n}\right)=z\right\} .
$$

A useful, but obvious property of $C(f, P)$ is the following:

Lemma 1.6. Let $D \subset \mathbb{C}$ be a domain and $f: D \rightarrow \mathbb{C} \cup\{\infty\}$ be continuous on $D$. If $D$ is locally connected at $P \in \bar{D}$, then $C(f, P)$ is either one point, or a continuum.

(This result appears in [10], as Theorem 1.1.)

The first one is the following theorem of Seidel, which describes the behavior of certain analytic functions near the boundary of their domain of definition. For proof, we refer to [10], Theorem 5.4.

Theorem 1.7. Let $f: \mathbb{D} \rightarrow \mathbb{D}$ be an analytic function such that the radial limit $f\left(\mathrm{e}^{\mathrm{i} \theta}\right)=\lim _{r \rightarrow 1} f\left(r \mathrm{e}^{\mathrm{i} \theta}\right)$ has modulus 1 for almost every $\theta \in\left(\theta_{1}, \theta_{2}\right)$. If $\theta \in\left(\theta_{1}, \theta_{2}\right)$ is such that $f$ does not extend analytically through $e^{\mathrm{i} \theta}$, then $C\left(f, \mathrm{e}^{\mathrm{i} \theta}\right)=\overline{\mathbb{D}}$.

The second result is the following theorem of Carathéodory (Theorem 5.5 in [10]):

Theorem 1.8. Let $f(z)$ be analytic and bounded in $|z|<1$. Assume that for almost every $\theta \in\left(\theta_{1}, \theta_{2}\right)$ the radial limit $f\left(\mathrm{e}^{\mathrm{i} \theta}\right)$ belongs to a set $W$ in the plane. Then, for every $\theta \in\left(\theta_{1}, \theta_{2}\right)$ the cluster set $C\left(f, \mathrm{e}^{\mathrm{i} \theta}\right)$ is contained in the closed convex hull of $W$.

Theorem 1.7 can be applied to self-maps of the upper half-plane $\mathbb{C}^{+}$, via a conformal mapping, but in that case one must consider meromorphic, instead of analytic, extensions.

Theorems 1.7 and 1.8 allow us to prove the following

\section{Proposition 1.9.}

(a) Let $f$ be an analytic self-map of $\mathbb{D}$ such that $\left|\lim _{r \rightarrow 1} f\left(r \mathrm{e}^{\mathrm{i} \theta}\right)\right|=1$ for almost every $\theta \in\left(\theta_{1}, \theta_{2}\right)$. Suppose that there is a point $\theta_{0} \in\left(\theta_{1}, \theta_{2}\right)$ such that the function $f$ cannot be continued analytically through $\mathrm{e}^{\mathrm{i} \theta}$. Then for any $t_{1}<t_{2}$ there is a set $E \subset\left(\theta_{1}, \theta_{2}\right)$ of nonzero Lebesgue measure such that $\lim _{r \rightarrow 1} f\left(r \mathrm{e}^{\mathrm{i} \theta}\right)$ exists for all $\theta \in E$, and the set $\left\{\lim _{r \rightarrow 1} f\left(r \mathrm{e}^{\mathrm{i} \theta}\right): \theta \in E\right\}$ is dense in the $\operatorname{arc} A=\left\{\mathrm{e}^{\mathrm{i} t}: t_{1}<t<t_{2}\right\}$.

(b) Let $f$ be an analytic self-map of $\mathbb{C}^{+}$such that $\lim _{y \rightarrow 0} f(x+\mathrm{i} y)$ exists and belongs to $\mathbb{R}$ for almost every $x \in(a, b)$. Suppose that $x_{0} \in(a, b)$ is such that $f$ cannot be continued meromorphically through $x_{0}$. Then for any $c<d$ there is a set $E \subset(a, b)$ of nonzero Lebesgue measure such that $\lim _{y \rightarrow 0} f(x+\mathrm{i} y)$ exists for all $x \in E$, and the set $\left\{\lim _{y \rightarrow 0} f(x+\mathrm{i} y): x \in E\right\}$ is dense in the interval $(c, d)$. 
Proof. Let $f$ and $\theta_{0}$ be as in the hypothesis of (a). Using Theorems 1.7 and 1.8 , we conclude that, on the one hand, $C\left(f, \mathrm{e}^{\mathrm{i} \theta_{0}}\right)=\overline{\mathbb{D}}$, and on the other, that $C\left(f, \mathrm{e}^{\mathrm{i} \theta_{0}}\right)$ equals the closure of the convex hull of the set $\left\{\lim _{r \rightarrow 1} f\left(r \mathrm{e}^{\mathrm{i} \theta}\right): \theta \in G\right\}$ for any set $G \subset\left(\theta_{1}, \theta_{2}\right)$ with the property that $\lim _{r \rightarrow 1} f\left(r \mathrm{e}^{\mathrm{i} \theta}\right)$ exists for all $\theta \in G$, and $\left(\theta_{1}, \theta_{2}\right) \backslash G$ has zero linear measure. This implies that the set

$$
\left\{\lim _{r \rightarrow 1} f\left(r \mathrm{e}^{\mathrm{i} \theta}\right): \theta_{1}<\theta<\theta_{2}, \lim _{r \rightarrow 1} f\left(r \mathrm{e}^{\mathrm{i} \theta}\right) \text { exists and belongs to } A\right\}
$$

is dense in $A$. It remains to prove that the set $E$ of those $\theta \in\left(\theta_{1}, \theta_{2}\right)$ such that $\lim _{r \rightarrow 1} f\left(r \mathrm{e}^{\mathrm{i} \theta}\right)$ exists and belongs to $A$ has nonzero linear measure. If this were not true, then, according to Theorem 1.8, we could take $G \backslash E$ instead of $G$ in the previous argument, and obtain a contradiction. This proves (a).

Part (b) follows directly from (a), by using the conformal mapping $z \mapsto(z-\mathrm{i}) /(z+\mathrm{i})$ and its inverse.

Consider now a Borel probability measure $\mu$ on $\mathbb{R}$. It is a well-known fact that one can draw informations about a measure from the behavior of its Poisson integral near the support of that measure. For example, the value at $x \in \mathbb{R}$ of the density function of the absolutely continuous part with respect to the Lebesgue measure of a finite measure can be obtained as nontangential limit at $x$ of the Poisson integral of the measure for almost all $x \in \mathbb{R}$ (for more details we refer to [13]). A simple computation shows that the imaginary part of the Cauchy transform of $\mu$ is (up to a multiple of $-\pi$ ) equal to the Poisson integral of $\mu$ :

$$
\Im G_{\mu}(x+\mathrm{i} y)=-\int_{\mathbb{R}} \frac{y}{(x-t)^{2}+y^{2}} \mathrm{~d} \mu(t), \quad x \in \mathbb{R}, y>0 .
$$

On the other hand, the reciprocal of the Cauchy transform of $\mu, F_{\mu}$, maps $\mathbb{C}^{+}$into itself. Thus, it will be natural to investigate the boundary behavior of the analytic map $F_{\mu}$ (and, implicitly, of $G_{\mu}$ ) in order to draw conclusions about $\mu$.

The following result is well known. Since we do not know any reference, we give here a full proof. We do not claim any paternity of this proof.

Lemma 1.10. Let $\mu$ be a Borel probability measure on $\mathbb{R}$, and denote by $\mu^{\mathrm{sc}}$ its singular continuous part. Then, for $\mu^{\mathrm{sc}}$-almost all $x \in \mathbb{R}$, the nontangential limit of the imaginary part of the Cauchy transform $G_{\mu}$ of $\mu$ at $x$ is infinite.

Proof. According to Theorem 1.4, it is enough to show that, for $\mu^{\mathrm{sc}}$-almost all $x \in \mathbb{R}$, the imaginary part of $G_{\mu}(x+\mathrm{i} y)$ tends to infinity as $y$ approaches zero. As we have seen before, for any $y>0$ and $x \in \mathbb{R}$,

$$
-\Im G_{\mu}(x+\mathrm{i} y)=\int_{\mathbb{R}} \frac{y}{(x-t)^{2}+y^{2}} \mathrm{~d} \mu(t) .
$$

Let us observe that $y^{2} /\left((x-t)^{2}+y^{2}\right) \geqslant 1 / 2$ for all $t \in \mathbb{R}$ such that $|x-t| \leqslant y$. Thus, for any given $y>0$, the following holds:

$$
\begin{aligned}
-\Im G_{\mu}(x+\mathrm{i} y) & =\int_{\mathbb{R}} \frac{y}{(x-t)^{2}+y^{2}} \mathrm{~d} \mu(t)=\frac{1}{y} \int_{\mathbb{R}} \frac{y^{2}}{(x-t)^{2}+y^{2}} \mathrm{~d} \mu(t) \\
& \geqslant \frac{1}{y} \int_{x-y}^{x+y} \frac{y^{2}}{(x-t)^{2}+y^{2}} \mathrm{~d} \mu(t) \geqslant \frac{\mu^{\mathrm{sc}}((x-y, x+y])}{2 y} .
\end{aligned}
$$

Now we can apply de La Vallée Poussin's theorem (Theorem 9.6 in [12]) to conclude that $\lim _{y \rightarrow 0} \mu^{\mathrm{sc}}((x-y, x+y]) /$ $(2 y)=\infty$ for $\mu^{\text {sc }}$-almost all $x \in \mathbb{R}$ (see also Theorem 31.6 in [9]).

To study properties of the free multiplicative convolutions of probability measures, one finds more convenient to use the functions $\psi_{\mu}$ and $\eta_{\mu}$ defined at the beginning of the introduction.

Remark 1.11. The formula $G_{\mu}(1 / z)=z\left(\psi_{\mu}(z)+1\right)$ together with Lemma 1.10 above, allows us to conclude that for $\mu^{\mathrm{sc}}$-almost all $x \in(0,+\infty)$, the nontangential limit of $\psi_{\mu}$ at $1 / x$ is infinite. 
Remark 1.12. For measures $\mu \in \mathcal{M}_{*}$, we have

$$
\psi_{\mu}(z)=\int_{\mathbb{T}} \frac{z}{\bar{t}-z} \mathrm{~d} \mu(t)=-\frac{1}{2}+\frac{1}{2} \int_{\mathbb{T}} \frac{t+z}{t-z} \mathrm{~d} \mu(\bar{t}), \quad z \in \mathbb{D},
$$

and hence the function $\psi_{\mu}$ satisfies the condition $\Re \psi_{\mu}(z) \geqslant-1 / 2$ for all $z \in \mathbb{D}$. The real part of

$$
\frac{1}{\pi}\left(\psi_{\mu}(z)+\frac{1}{2}\right)=\frac{1}{2 \pi} \int_{\mathbb{T}} \frac{t+z}{t-z} \mathrm{~d} \mu(\bar{t}), \quad z \in \mathbb{D},
$$

is the Poisson integral of the measure $\mathrm{d} \mu(\bar{t})$, and thus, by Lemma 1.10 and a conformal transformation, for $\mu^{\text {sc }}$-almost all $x \in \mathbb{T}$, the nontangential limit of $\psi_{\mu}$ at $\bar{x}$ is infinite (a detailed presentation of Poisson integrals of measures on the unit circle can be found in [11]).

We conclude the introduction with a proposition which essentially characterizes analytic functions that are Cauchy transforms of probability measures.

\section{Proposition 1.13.}

(a) For every $\mu \in \mathcal{M}$, we have $\Im F_{\mu}(z) \geqslant \Im z, z \in \mathbb{C}^{+}$. The inequality is strict if and only if $\mu$ is not a point mass.

(b) For every $\mu \in \mathcal{M}_{+}, \mu \neq \delta_{0}$, we have $\pi>\arg \eta_{\mu}(z) \geqslant \arg z, z \in \mathbb{C}^{+}$. The second inequality is strict if and only if $\mu$ is not a point mass.

(c) For every $\mu \in \mathcal{M}_{*}$, we have $\left|\eta_{\mu}(z)\right| \leqslant|z|, z \in \mathbb{D}$. The inequality is strict if and only if $\mu$ is not a point mass.

Part (a) of the above proposition can be found also in [5], while parts (b) and (c) appear in [3] as Propositions 2.2 and 3.2.

\section{Boundary behavior of $G_{\mu \boxplus v}$}

In the following we shall denote by $\operatorname{supp} \tau$ the topological support of the measure $\tau$, and by $\tau^{\text {ac }}$ (respectively $\tau^{\mathrm{s}}, \tau^{\mathrm{sc}}, \tau^{\mathrm{a}}$ ) the absolutely continuous (respectively singular, singular continuous, atomic) part of the measure $\tau$ with respect to the Lebesgue measure. We shall fix two Borel probability measures $\mu, v$ on the real line, none of them concentrated in one point. For simplicity, denote the subordination functions $\omega_{\mu, \nu}$ and $\omega_{\nu, \mu}$ provided by Theorem 1.1 by $\omega_{1}$ and $\omega_{2}$, respectively.

Lemma 2.1 ([4]). Assume that there exists $a \in \mathbb{R}$ such that $F_{\mu}(z)=F_{\nu}\left(a+F_{\mu}(z)-z\right)$ for all $z \in \mathbb{C}^{+}$. Then the functions $h_{\mu}(z)=F_{\mu}(z)-z+a, h_{v}(z)=F_{\nu}(z)-z+a, z \in \mathbb{C}^{+}$, are conformal self-maps of the upper half-plane, inverse to each other.

Proof. Let $h_{\mu}, h_{\nu}$ be as in the statement of the lemma. We have:

$$
z=F_{v}\left(F_{\mu}(z)-z+a\right)-\left(F_{\mu}(z)-z+a\right)+a=h_{v}\left(h_{\mu}(z)\right), \quad z \in \mathbb{C}^{+} .
$$

Since, by Proposition 1.13, both $h_{\mu}$ and $h_{\nu}$ are nonconstant analytic self-maps of the upper half-plane, applying $h_{\mu}$ in both sides of the previous equality yields $h_{\mu}\left(h_{v}(w)\right)=w$, for all $w$ in the open nonempty set $h_{\mu}\left(\mathbb{C}^{+}\right)$, and, by analytic continuation, for all $w \in \mathbb{C}^{+}$. This shows that both $h_{\mu}, h_{v}$ are conformal self-maps of $\mathbb{C}^{+}$inverse to each other and completes the proof.

We effectively use in the proof the fact that neither one of $\mu, v$ is concentrated at one point.

Remark 2.2. Any function $F_{\mu}$ satisfying the conditions of Lemma 2.1 must be of the form

$$
F_{\mu}(z)=\frac{z^{2}+m z+p}{z+r}, \quad m, p, r \in \mathbb{R},
$$


and thus, $\mu$ is purely atomic with at most two atoms. Using relation (1), it is easy to see that, if both $\mu$ and $v$ are convex combinations of two atoms, then for any $x \in \mathbb{R} \lim _{z \rightarrow x, \Im z>0} F_{\mu \boxplus v}(z)$ exists in $\mathbb{C}^{+} \cup \mathbb{R} \cup\{\infty\}$.

The next theorem describes the boundary behavior of the Cauchy transform of $\mu \boxplus \nu$.

Theorem 2.3. Let $\mu, v \in \mathcal{M}$, none of them concentrated in one point. Then the set

$$
\mathcal{C}_{+}=\left\{a \in \mathbb{R}: C\left(F_{\mu \boxplus v}, a\right) \cap \mathbb{C}^{+} \text {is infinite }\right\}
$$

is empty. If $\mu$ and $v$ have compact support, then the set

$$
\mathcal{C}=\left\{a \in \mathbb{R}: C\left(F_{\mu \boxplus v}, a\right) \text { is infinite }\right\}
$$

is empty.

Let us note that $\mathcal{C}$ is empty if and only if $\lim _{z \rightarrow x, \Im z>0} G_{\mu \boxplus v}(z)$ exists for all $x \in \mathbb{R}$, or, equivalently, if the restriction of $G_{\mu \boxplus v}$ to the upper half-plane extends continuously to $\mathbb{C}^{+} \cup \mathbb{R}$ as function with values in $\mathbb{C} \cup\{\infty\}$.

Proof. We shall consider the restriction of the functions $F$ and $\omega$ to the upper half-plane. By Lemma 2.1 and Remark 2.2, it is enough to show that if $a \in \mathcal{C}_{+}$(or, in the second case, if $a \in \mathcal{C}$ ), then $F_{\mu}$ and $F_{\nu}$ satisfy either $F_{\mu}(z)=F_{v}\left(a+F_{\mu}(z)-z\right)$, or $F_{v}(z)=F_{\mu}\left(a+F_{v}(z)-z\right)$.

Suppose $a \in \mathcal{C}_{+}$. The relation

$$
\omega_{1}(z)+\omega_{2}(z)=z+F_{\mu \boxplus v}(z)
$$

from Lemma 1.2(a), together with $G_{\mu} \circ \omega_{1}=G_{\nu} \circ \omega_{2}=G_{\mu \boxplus \nu}$, assures us that at least one of $C\left(\omega_{1}, a\right) \cap \mathbb{C}^{+}$, $C\left(\omega_{2}, a\right) \cap \mathbb{C}^{+}$will be infinite. Without loss of generality, assume that $C\left(\omega_{1}, a\right) \cap \mathbb{C}^{+}$is infinite, hence, according to Lemma 1.6, a continuum.

For any $z \in C\left(\omega_{1}, a\right) \cap \mathbb{C}^{+}$there exists a sequence $\left\{z_{n}\right\}_{n \in \mathbb{N}} \subset \mathbb{C}^{+}$converging to $a$ such that $\lim _{n \rightarrow \infty} \omega_{1}\left(z_{n}\right)=z$. Using Lemma 1.2(a), we have:

$$
\lim _{n \rightarrow \infty} \omega_{2}\left(z_{n}\right)=\lim _{n \rightarrow \infty} z_{n}+F_{\mu \boxplus v}\left(z_{n}\right)-\omega_{1}\left(z_{n}\right)=a+\lim _{n \rightarrow \infty} F_{\mu}\left(\omega_{1}\left(z_{n}\right)\right)-z=a+F_{\mu}(z)-z .
$$

Now the subordination formula from Theorem 1.1(a), will give

$$
F_{\mu}(z)=\lim _{n \rightarrow \infty} F_{\mu}\left(\omega_{1}\left(z_{n}\right)\right)=\lim _{n \rightarrow \infty} F_{\nu}\left(\omega_{2}\left(z_{n}\right)\right)=F_{v}\left(a+F_{\mu}(z)-z\right)
$$

for all $z \in C\left(\omega_{1}, a\right) \cap \mathbb{C}^{+}$. Since $C\left(\omega_{1}, a\right) \cap \mathbb{C}^{+}$, is a continuum in $\mathbb{C}^{+}$, the identity theorem for analytic functions will imply that

$$
F_{\mu}(z)=F_{\nu}\left(a+F_{\mu}(z)-z\right)
$$

for all $z \in \mathbb{C}^{+}$. By Remark 2.2, we have $\mathcal{C}_{+}=\emptyset$.

Let us assume now that $\mu$ and $v$ have compact support, and suppose that $C\left(F_{\mu \boxplus v}, a\right) \subseteq \mathbb{R} \cup\{\infty\}$ has more than one point. Then, by Lemma 1.6, $C\left(F_{\mu \boxplus v}, a\right)$ must be either a closed interval, or the complement of an open (possibly empty) interval in $\mathbb{R} \cup\{\infty\}$. It is easy to see that $C\left(\omega_{1}, a\right), C\left(\omega_{2}, a\right)$ do not contain points in $\mathbb{C}^{+}$. As before, at least one of $C\left(\omega_{1}, a\right), C\left(\omega_{2}, a\right) \subseteq \mathbb{R} \cup\{\infty\}$ will be nontrivial. Without loss of generality, assume $C\left(\omega_{1}, a\right)$ is nontrivial.

We claim that for any $c$ in $C\left(\omega_{1}, a\right) \backslash\{\infty\}$, with the possible exception of three points, there exists a sequence $\left\{z_{n}^{(c)}\right\}_{n \in \mathbb{N}}$ converging to $a$ such that $\lim _{n \rightarrow \infty} \omega_{1}\left(z_{n}^{(c)}\right)=c$, and $\Re \omega_{1}\left(z_{n}^{(c)}\right)=c$ for all $n$. Let $\left\{c_{n}\right\}_{n \in \mathbb{N}}$ be a dense sequence in $C\left(\omega_{1}, a\right)$, and consider $z_{n} \in \mathbb{C}^{+}$, such that $\left|z_{n}-a\right|<1 / n$, and $\left|\omega_{1}\left(z_{n}\right)-c_{n}\right|<1 / n, n \in \mathbb{N}$. We define a path $\gamma:[0,1] \rightarrow \mathbb{C}^{+} \cup\{a\}$ such that $\gamma(1-1 / n)=z_{n}, \gamma(1)=a$, and $\gamma$ is linear on the intervals $[1-1 / n, 1-1 /(n+1)]$, $n \in \mathbb{N}$. It will suffice to show that there exists at most one point $c$ in the interior of $C\left(\omega_{1}, a\right)$ such that $\omega_{1}(\gamma([0,1))) \cap$ $\{c+\mathrm{i} t: t \in[0, \varepsilon)\}=\emptyset$ for some $\varepsilon>0$. Indeed, assume to the contrary that $c<c^{\prime}$ are two such points. The set

$$
K=\{c+\mathrm{i} t: t \in(0,1)\} \cup\left\{c^{\prime}+\mathrm{i} t: t \in(0,1)\right\} \cup\left\{s+\mathrm{i}, c \leqslant s \leqslant c^{\prime}\right\}
$$

separates $\mathbb{C}^{+}$into two components, and the path $\omega_{1}(\gamma(t))$ contains infinitely many points in either of the components, hence it crosses $K$ infinitely many times. By our assumption, crossings cannot be close to $c$ or $c^{\prime}$, and this implies the existence of a point in $C\left(\omega_{1}, a\right) \cap K \subset \mathbb{C}^{+}$, contradicting the fact that $C\left(\omega_{1}, a\right) \cap \mathbb{C}^{+}=\emptyset$. This proves our claim. 
We shall next show that, in fact, both $C\left(\omega_{1}, a\right), C\left(\omega_{2}, a\right) \subseteq \mathbb{R} \cup\{\infty\}$ contain more than one point. Indeed, suppose, for example, that $\lim _{z \rightarrow a} \omega_{2}(z)=\omega_{2}(a) \in \mathbb{R} \cup\{\infty\}$. If $\omega_{2}(a)=\infty$, then

$$
-\int_{-\infty}^{\infty} t \mathrm{~d} v(t)=\lim _{z \rightarrow \infty} F_{v}(z)-z=\lim _{z \rightarrow a} F_{v}\left(\omega_{2}(z)\right)-\omega_{2}(z)=\lim _{z \rightarrow a} \omega_{1}(z)-z,
$$

so that $C\left(\omega_{1}, a\right)=\left\{a-\int_{-\infty}^{\infty} t \mathrm{~d} v(t)\right\}$, which is a contradiction. Assume $\omega_{2}(a) \in \mathbb{R}$. By Theorems $1.3,1.4$, and 1.5, the nontangential limit of $F_{\mu}$ at $x$ exists and is finite for all $x \in \mathbb{R}$, with the possible exception of a subset of Lebesgue measure zero. Thus, for almost all $c \in C\left(\omega_{1}, a\right)$, we have:

$$
\begin{aligned}
F_{\mu}(c) & =\lim _{z \vec{\varangle}^{c}} F_{\mu}(z)=\lim _{n \rightarrow \infty} F_{\mu}\left(\omega_{1}\left(z_{n}^{(c)}\right)\right)=\lim _{n \rightarrow \infty} F_{\mu \boxplus \nu}\left(z_{n}^{(c)}\right)=\lim _{n \rightarrow \infty} \omega_{1}\left(z_{n}^{(c)}\right)+\omega_{2}\left(z_{n}^{(c)}\right)-z_{n}^{(c)} \\
& =c+\omega_{2}(a)-a .
\end{aligned}
$$

Using Privalov's theorem (Theorem 1.5), we conclude that $F_{\mu}(z)=z-\left(a-\omega_{2}(a)\right)$ for all $z \in \mathbb{C}^{+}$. This contradicts the fact that $\mu$ is not concentrated at the point $a-\omega_{2}(a)$. So indeed both $C\left(\omega_{1}, a\right), C\left(\omega_{2}, a\right)$ are infinite.

Assume that the nontangential limit of $F_{\mu}$ exists at $c \in C\left(\omega_{1}, a\right)$, and there exists a sequence $\left\{z_{n}^{(c)}\right\}_{n \in \mathbb{N}}$ converging to $a$ such that $\lim _{n \rightarrow \infty} \omega_{1}\left(z_{n}^{(c)}\right)=c$ and $\Re \omega_{1}\left(z_{n}^{(c)}\right)=c$ for all $n$. Then

$$
F_{\mu}(c)=\lim _{z \mathbb{\varangle}^{c}} F_{\mu}(z)=\lim _{n \rightarrow \infty} F_{\mu}\left(\omega_{1}\left(z_{n}^{(c)}\right)\right)=\lim _{n \rightarrow \infty} F_{\mu \boxplus v}\left(z_{n}^{(c)}\right)=\lim _{n \rightarrow \infty} F_{v}\left(z_{n}^{(c)}+F_{\mu}\left(\omega_{1}\left(z_{n}^{(c)}\right)\right)-\omega_{1}\left(z_{n}^{(c)}\right)\right) .
$$

If there exists a subset $E$ of $C\left(\omega_{1}, a\right)$ of nonzero Lebesgue measure such that $F_{\mu}(c) \in \mathbb{C}^{+}$for all $c \in E$, then we have

$$
F_{\mu}(c)=\lim _{z \underset{\varangle}{ } \vec{\varangle}^{c}} F_{\mu}(z)=\lim _{z \mathbb{\varangle}^{c}} F_{\nu}\left(a+F_{\mu}(z)-z\right)=F_{\nu}\left(a+F_{\mu}(c)-c\right), \quad c \in E .
$$

By Theorem 1.5, this implies that the equality $F_{\mu}(z)=F_{\nu}\left(a+F_{\mu}(z)-z\right)$ holds for all $z \in \mathbb{C}^{+}$, and thus, by Remark 2.2, we obtain a contradiction.

If $F_{\mu}(c) \in \mathbb{R}$ for almost all $c \in C\left(\omega_{1}, a\right)$, then we use Theorem 1.7 to conclude that either $C\left(F_{\mu}, c\right)=\mathbb{C}^{+} \cup \mathbb{R} \cup\{\infty\}$ for some $c \in C\left(\omega_{1}, a\right)$, or $F_{\mu}$ extends meromorphically through the interior of $C\left(\omega_{1}, a\right)$.

In the former case, since supp $v$ is compact, we use Proposition 1.9(b) to conclude that there exists a set $E \subset$ $C\left(\omega_{1}, a\right)$ of nonzero Lebesgue measure such that

$$
\lim _{z \gtrless^{c}} F_{\mu}(z)-z+a \in \mathbb{R} \backslash \operatorname{supp} v, \quad c \in E .
$$

But this means that

$$
F_{\mu}(c)=\lim _{z \underset{\varangle}{\mathbb{*}}} F_{\mu}(z)=\lim _{n \rightarrow \infty} F_{\mu}\left(\omega_{1}\left(z_{n}^{(c)}\right)\right)=\lim _{n \rightarrow \infty} F_{\nu}\left(z_{n}^{(c)}+F_{\mu}\left(\omega_{1}\left(z_{n}^{(c)}\right)\right)-\omega_{1}\left(z_{n}^{(c)}\right)\right)=F_{\nu}\left(a+F_{\mu}(c)-c\right),
$$

for all $c \in E$. Since $E$ is of positive Lebesgue measure, we apply Privalov's theorem to conclude that $F_{\mu}(z)=F_{\nu}(a+$ $\left.F_{\mu}(z)-z\right)$ for all $z \in \mathbb{C}^{+}$and obtain a contradiction.

In the latter case, we look at $C\left(\omega_{2}, a\right)$. If either there is a subset of nonzero Lebesgue measure of $C\left(\omega_{2}, a\right)$ on which the nontangential limit of $F_{v}$ has imaginary part strictly greater than zero, or there is a $c^{\prime} \in C\left(\omega_{2}, a\right)$ at which $C\left(F_{v}, c^{\prime}\right)=\mathbb{C}^{+} \cup \mathbb{R} \cup\{\infty\}$, then by the same argument as before we have the equality

$$
F_{\nu}(z)=F_{\mu}\left(a+F_{\nu}(z)-z\right), \quad z \in \mathbb{C}^{+},
$$

and then $C\left(F_{\mu \boxplus \nu}, a\right)$ must be trivial by Lemma 2.1 and Remark 2.2.

The only case that remains to be analyzed is when $F_{\mu}$ extends analytically through the interior of $C\left(\omega_{1}, a\right)$ and $F_{v}$ extends analytically through the interior of $C\left(\omega_{2}, a\right)$. By dropping if necessary to a subsequence, we may assume that both $c=\lim _{n \rightarrow \infty} \omega_{1}\left(z_{n}^{(c)}\right)$ and $\lim _{n \rightarrow \infty} \omega_{2}\left(z_{n}^{(c)}\right)$ exist, where $z_{n}^{(c)}$ is such that $\Re \omega_{1}\left(z_{n}^{(c)}\right)=c$.

Suppose there were a point $d \in C\left(\omega_{2}, a\right)$ and a set $V_{d} \subseteq C\left(\omega_{1}, a\right)$ of nonzero Lebesgue measure such that $\lim _{n \rightarrow \infty} \omega_{2}\left(z_{n}^{(c)}\right)=d$ for all $c \in V_{d}$. Taking limit as $n \rightarrow \infty$ in the equality

$$
F_{\mu}\left(\omega_{1}\left(z_{n}^{(c)}\right)\right)+z_{n}^{(c)}=F_{\mu \boxplus v}\left(z_{n}^{(c)}\right)+z_{n}^{(c)}=\omega_{1}\left(z_{n}^{(c)}\right)+\omega_{2}\left(z_{n}^{(c)}\right)
$$


gives

$$
F_{\mu}(c)+a=c+d, \quad \text { for all } c \in V_{d} .
$$

Applying again Privalov's theorem, we obtain that $F_{\mu}(z)=z-(a-d)$ for all $z \in \mathbb{C}^{+}$. This contradicts the fact that $\mu$ is not concentrated at the point $a-d$. Thus, there exists a set $E \subset C\left(\omega_{1}, a\right)$ of positive Lebesgue measure such that $\left\{c^{\prime}=\lim _{n \rightarrow \infty} \omega_{2}\left(z_{n}^{(c)}\right): c \in E\right\} \subseteq \operatorname{Int} C\left(\omega_{2}, a\right)$. Then

$$
\begin{aligned}
F_{\mu}(c) & =\lim _{z \rightarrow c} F_{\mu}(z)=\lim _{n \rightarrow \infty} F_{\mu}\left(\omega_{1}\left(z_{n}^{(c)}\right)\right)=\lim _{n \rightarrow \infty} F_{\mu \boxplus v}\left(z_{n}^{(c)}\right)=\lim _{n \rightarrow \infty} F_{\nu}\left(\left(z_{n}^{(c)}+F_{\mu}\left(\omega_{1}\left(z_{n}^{(c)}\right)\right)-\omega_{1}\left(z_{n}^{(c)}\right)\right)\right. \\
& =F_{\nu}\left(a+F_{\mu}(c)-c\right)
\end{aligned}
$$

for all $c \in E$. Privalov's theorem implies that $F_{\mu}(z)=F_{v}\left(a+F_{\mu}(z)-z\right)$ for all $z \in \mathbb{C}^{+}$. This concludes the proof.

Remark 2.4. The proof of Theorem 2.3 implies that, whenever $\mu, v$ have compact support and neither of them is concentrated in one point, the restrictions to the upper half-plane of $\omega_{1}$ and $\omega_{2}$ can be continuously extended to $\mathbb{R}$.

An immediate consequence of Theorem 2.3 is the following

Corollary 2.5. If $\mu$ and $v$ have compact support and neither of them is concentrated in one point, then $\operatorname{supp}(\mu \boxplus v)^{\mathrm{s}}$ is closed and of zero Lebesgue measure. Moreover, $\operatorname{supp}(\mu \boxplus v)^{\mathrm{sc}} \subset \operatorname{supp}(\mu \boxplus v)^{\mathrm{ac}}$. In particular, $\mu \boxplus v$ can never be singular (the last statement holds also for Borel probability measures with noncompact support on $\mathbb{R}$ ).

Proof. Suppose first that $\mu, v$ have compact support. According to Theorem 2.3, $F_{\mu \boxplus v}$ extends continuously to $\mathbb{R}$. Thus, the preimage of zero under the extension of $F_{\mu \boxplus v}$ to $\mathbb{C}^{+} \cup \mathbb{R}$ must be a closed set. This fact, together with Lemma 1.10, imply that the set $\operatorname{supp}(\mu \boxplus v)^{\mathrm{sc}}$ is included in the closed set $F_{\mu \boxplus \nu}^{-1}(\{0\})$. According to Privalov’s theorem (Theorem 1.5), $F_{\mu \boxplus v}^{-1}(\{0\})$ must be of zero Lebesgue measure. Since by Theorem 7.4 of [6], $\mu \boxplus v$ can have only finitely many atoms, we conclude that $\operatorname{supp}(\mu \boxplus v)^{\mathrm{s}}$ is a closed set of zero Lebesgue measure.

Assume now that $x \in \operatorname{supp}(\mu \boxplus v)^{\mathrm{sc}}$. As $\mu \boxplus v$ can have only finitely many atoms, there is no loss of generality to assume that $x$ is not an atom of $\mu \boxplus \nu$. Recall that $-\pi^{-1} \Im G_{\mu \boxplus v}$ is the Poisson integral of $\mu \boxplus v$. Assume to the contrary that $x$ does not belong to $\operatorname{supp}(\mu \boxplus v)^{\mathrm{ac}}$. Thus, there exists an open neighborhood $V \subseteq \mathbb{R}$ of $x$ such that the nontangential limit of the Poisson integral of $\mu \boxplus v$ at $a$ equals zero for almost all $a \in V$ with respect to the Lebesgue measure. We conclude that $\varangle \lim _{z \rightarrow a} G_{\mu \boxplus v}(z) \in \mathbb{R}$ for Lebesgue-almost all $a \in V$. On the other hand, the set $V \cap \operatorname{supp}(\mu \boxplus v)^{\mathrm{sc}}$ is infinite, without isolated points, and the nontangential limit of $G_{\mu \boxplus v}$ at $(\mu \boxplus v)^{\mathrm{sc}}$-almost all points in $V \cap \operatorname{supp}(\mu \boxplus v)^{\mathrm{sc}}$ is infinite, by Lemma 1.10. Thus, the identity theorem for analytic functions implies that $G_{(\mu \boxplus v)}$ sc does not extend meromorphically through any $a \in V \cap \operatorname{supp}(\mu \boxplus v)^{\mathrm{sc}}$. We conclude by Theorem 1.7, applied to the upper half-plane, that $C\left(-G_{\mu \boxplus v}, x\right)=\mathbb{C}^{+} \cup \mathbb{R} \cup\{\infty\}$, and thus $C\left(F_{\mu \boxplus \nu}, x\right)=\mathbb{C}^{+} \cup \mathbb{R} \cup\{\infty\}$. This contradicts Theorem 2.3.

Finally, suppose that $\mu$ and $v$ have arbitrary support, and assume that $\mu \boxplus v$ is purely singular. According to Theorem 7.4 of [6], $\mu \boxplus v$ cannot be purely atomic, and can have only finitely many atoms. Thus, it must have a singular continuous component. As before, we conclude that for any $x \in \operatorname{supp}(\mu \boxplus v)$ which is not an atom of $\mu \boxplus v$ we have $C\left(F_{\mu \boxplus v}, x\right)=\mathbb{C}^{+} \cup \mathbb{R} \cup\{\infty\}$, contradicting Theorem 2.3.

\section{Boundary behavior of $\psi_{\mu \bowtie v}$}

\subsection{Measures supported on $[0,+\infty)$}

As in Section 2, let $\mu, v \in \mathcal{M}_{+}$, neither of them a point mass, and denote the subordination functions provided by Theorem $1.1 \omega_{\mu, \nu}=\omega_{1}$, and $\omega_{v, \mu}=\omega_{2}$. We have the following analogue of Lemma 2.1:

Lemma 3.1. If there exists $a \in(0,+\infty)$ such that $\eta_{\mu}(z)=\eta_{\nu}\left(\frac{a}{z} \eta_{\mu}(z)\right)$, then there exist $\alpha_{1}, \alpha_{2}, \beta_{1}, \beta_{2}>0, t, s \in(0,1)$ such that $\alpha_{1} \neq \alpha_{2}, \beta_{1} \neq \beta_{2}$, and $\mu=t \delta_{\alpha_{1}}+(1-t) \delta_{\alpha_{2}}, v=s \delta_{\beta_{1}}+(1-s) \delta_{\beta_{2}}$. 
Proof. Consider the analytic functions $h_{\mu}(z)=a \eta_{\mu}(z) / z, h_{v}(z)=a \eta_{v}(z) / z, z \in \mathbb{C} \backslash[0,+\infty)$. The equation $\eta_{\mu}(z)=$ $\eta_{\nu}\left(\frac{a}{z} \eta_{\mu}(z)\right)$ implies

$$
h_{\nu}\left(h_{\mu}(z)\right)=\frac{a}{h_{\mu}(z)} \eta_{\nu}\left(h_{\mu}(z)\right)=\frac{a}{h_{\mu}(z)} \eta_{\mu}(z)=z, \quad z \in \mathbb{C} \backslash \mathbb{R} .
$$

Since, by Proposition $1.13, h_{\mu}$ is not constant, applying $h_{\mu}$ to the previous equality, we obtain $h_{\mu}\left(h_{v}(w)\right)=w$ for all $w$ in the nonempty open set $h_{\mu}(\mathbb{C} \backslash \mathbb{R})$. So $h_{\mu}$ and $h_{v}$ are conformal maps inverse to each other.

Since $z\left(\psi_{\mu}(z)+1\right)=G_{\mu}(1 / z)$, we have

$$
h_{\mu}(z)=a\left(\frac{1}{z}-\frac{1}{G_{\mu}(1 / z)}\right)=a\left(\frac{1}{z}-F_{\mu}\left(\frac{1}{z}\right)\right), \quad z \in \mathbb{C} \backslash[0,+\infty),
$$

so $h_{\mu}\left(\mathbb{C}^{+}\right) \subseteq \mathbb{C}^{+}$, by Proposition 1.13 .

As we also have $\eta_{\mu}((-\infty, 0)) \subset(-\infty, 0)$, so that $h_{\mu}$ maps $(-\infty, 0)$ into $(0, \infty)$, and $\mu, v$ are not point masses, it follows that

$$
h_{\mu}(z)=\frac{c z+d}{z+b}, \quad c>0, b<0, d<c b, z \neq-b .
$$

Then

$$
h_{v}(z)=\frac{-b z+d}{z-c}, \quad z \neq c .
$$

Thus, $\eta_{\mu}(z)=\left(c z^{2}+d z\right) /(a z+a b)$ and $\eta_{v}(z)=\left(-b z^{2}+d z\right) /(a z-a c)$ extend analytically through $[0,+\infty) \backslash\{-b\}$, and $[0,+\infty) \backslash\{c\}$, respectively. This implies that both $\mu$ and $v$ must be convex combination of two distinct Dirac measures, $\mu=t \delta_{\alpha_{1}}+(1-t) \delta_{\alpha_{2}}$, and $v=s \delta_{\beta_{1}}+(1-s) \delta_{\beta_{2}}$ for some $\alpha_{1}, \alpha_{2}, \beta_{1}, \beta_{2}>0, t, s \in(0,1)$, and concludes the proof.

It is not obvious that, for $\mu$ and $v$ as in Lemma 3.1, $C\left(\eta_{\mu{ }_{v}}, x\right)$ is finite for all $x \in[0,+\infty)$. To prove this, we shall compute explicitly $\eta_{\mu \otimes_{v}}$. Let us note that

$$
\begin{aligned}
\eta_{\mu}(z) & =\frac{\psi_{\mu}(z)}{1+\psi_{\mu}(z)}=\frac{t z \alpha_{1}\left(1-z \alpha_{2}\right)+(1-t) z \alpha_{2}\left(1-z \alpha_{1}\right)}{\left(1-z \alpha_{1}\right)\left(1-z \alpha_{2}\right)+t z \alpha_{1}\left(1-z \alpha_{2}\right)+(1-t) z \alpha_{2}\left(1-z \alpha_{1}\right)} \\
& =\frac{z\left(t \alpha_{1}+(1-t) \alpha_{2}\right)-z^{2} \alpha_{1} \alpha_{2}}{1-z\left((1-t) \alpha_{1}+t \alpha_{2}\right)},
\end{aligned}
$$

so that $\alpha_{1} \alpha_{2}=-c /(a b), \alpha_{1}+\alpha_{2}=(d-a) /(a b)$, and $t=\left(1+b \alpha_{1}\right) /\left(b \alpha_{1}-b \alpha_{2}\right)$. This implies

$$
b=\frac{1}{(t-1) \alpha_{1}-t \alpha_{2}}, \quad c=\frac{a \alpha_{1} \alpha_{2}}{(1-t) \alpha_{1}+t \alpha_{2}}, \quad \text { and } \quad d=a+\frac{a\left(\alpha_{1}+\alpha_{2}\right)}{(t-1) \alpha_{1}-t \alpha_{2}} .
$$

Thus, $\alpha_{1}, \alpha_{2}, t$, and $a$ determine uniquely $b, c$, and $d$. For simplicity, we shall compute $\eta_{\mu \otimes_{v}}$ in terms of $b, c, d$. Using successively Theorem 1.1(c), Lemmas 3.1, and 1.2, we have

$$
\eta_{\mu}\left(\omega_{1}(z)\right)=\eta_{\nu}\left(\omega_{2}(z)\right)=\eta_{\mu}\left(a \frac{\eta_{\nu}\left(\omega_{2}(z)\right)}{\omega_{2}(z)}\right)=\eta_{\mu}\left(a \frac{\omega_{1}(z)}{z}\right), \quad z \in \mathbb{C} \backslash[0,+\infty) .
$$

Since $\eta_{\mu}(z)=\left(c z^{2}+d z\right) /(a z+a b)$, the previous equality will imply that the subordination function $\omega_{1}$ satisfies

$$
a c \omega_{1}(z)^{2}+b c(a+z) \omega_{1}(z)+b d z=0, \quad z \in \mathbb{C} \backslash[0,+\infty) .
$$

This, together with the condition $\omega_{1}((-\infty, 0)) \subseteq(-\infty, 0)$ (required by Theorem 1.1(c1)), shows that

$$
\omega_{1}(z)=\frac{-b c(a+z)-\sqrt{b^{2} c^{2}(a+z)^{2}-4 a b c d z}}{2 a c}, \quad z \in \mathbb{C} \backslash[0,+\infty) .
$$

A similar computation gives

$$
\omega_{2}(z)=\frac{b c(a+z)+\sqrt{b^{2} c^{2}(a+z)^{2}-4 a b c d z}}{2 a b}, \quad z \in \mathbb{C} \backslash[0,+\infty) .
$$

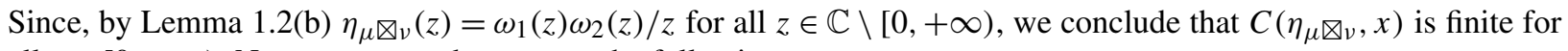
all $x \in[0,+\infty)$. Now we are ready to prove the following 
Theorem 3.2. Let $\mu, v \in \mathcal{M}_{+}$, none of them concentrated in one point. Then the set

$$
\mathcal{D}=\left\{a \in(0, \infty): C\left(\psi_{\mu \otimes_{\nu}}, a\right) \text { is infinite }\right\}
$$

is empty.

Let us note that $\mathcal{D}$ is empty if and only if $\lim _{z \rightarrow x, \Im z>0} \psi_{\mu 凶 v}(z)$ exists for all $x \in(0,+\infty)$, or, equivalently, if the restriction of $\psi_{\mu \bowtie \nu}$ to the upper half-plane extends continuously to $\mathbb{C}^{+} \cup \mathbb{R}$ as function with values in $\mathbb{C} \cup\{\infty\}$.

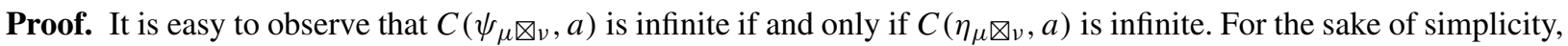
we will work mainly with $\eta$. As the functions $\psi, \eta$, and $\omega$ map the upper half-plane into itself, it will be convenient from now on to consider their restrictions to $\mathbb{C}^{+}$. We shall prove the theorem by showing that if $a \in \mathcal{D}$, then $\eta_{\mu}(z)=$ $\eta_{\nu}\left(a \eta_{\mu}(z) / z\right)$, and using Lemma 3.1 and the observations following it to obtain a contradiction. So suppose there exists a number $a \in \mathcal{D}$. The relation

$$
\eta_{\mu \bigotimes v}(z)=\frac{1}{z} \omega_{1}(z) \omega_{2}(z)
$$

from Lemma 1.2(b) assures us that at least one of $C\left(\omega_{1}, a\right), C\left(\omega_{2}, a\right)$ is infinite.

Consider first the case when $C\left(\eta_{\mu \otimes_{v}}, a\right) \cap \mathbb{C}^{+}$is infinite. Then at least one of $C\left(\omega_{1}, a\right) \cap \mathbb{C}^{+}, C\left(\omega_{2}, a\right) \cap \mathbb{C}^{+}$ is infinite. Without loss of generality, assume that $C\left(\omega_{1}, a\right) \cap \mathbb{C}^{+}$is infinite. For any $z \in C\left(\omega_{1}, a\right) \cap \mathbb{C}^{+}$, there is a sequence $\left\{z_{n}\right\}_{n \in \mathbb{N}}$ converging to $a$ such that $\lim _{n \rightarrow \infty} \omega_{1}\left(z_{n}\right)=z$. Then, by Lemma 1.2 and Theorem 1.1,

$$
\lim _{n \rightarrow \infty} \omega_{2}\left(z_{n}\right)=\lim _{n \rightarrow \infty} \frac{z_{n} \eta_{\mu} \otimes_{v}\left(z_{n}\right)}{\omega_{1}\left(z_{n}\right)}=\lim _{n \rightarrow \infty} \frac{z_{n} \eta_{\mu}\left(\omega_{1}\left(z_{n}\right)\right)}{\omega_{1}\left(z_{n}\right)}=\frac{a \eta_{\mu}(z)}{z} .
$$

Since $\mu$ is not a point mass, $\Im\left(\frac{a \eta_{\mu}(z)}{z}\right)>0$ whenever $\Im z>0$, by Proposition 1.13(b). This gives directly

$$
\eta_{\mu}(z)=\lim _{n \rightarrow \infty} \eta_{\mu}\left(\omega_{1}\left(z_{n}\right)\right)=\lim _{n \rightarrow \infty} \eta_{\nu}\left(\omega_{2}\left(z_{n}\right)\right)=\lim _{n \rightarrow \infty} \eta_{\nu}\left(\frac{z_{n} \eta_{\mu 凶 \nu}\left(z_{n}\right)}{\omega_{1}\left(z_{n}\right)}\right)=\eta_{\nu}\left(\frac{a \eta_{\mu}(z)}{z}\right),
$$

for all $z \in C\left(\omega_{1}, a\right) \cap \mathbb{C}^{+}$, and hence for all $z \in \mathbb{C} \backslash[0,+\infty)$, which, is a contradiction.

Now suppose that $C\left(\eta_{\mu \boxplus v}, a\right) \subseteq \mathbb{R} \cup\{\infty\}$ is infinite. By an argument similar to the one in the proof of Theorem 2.3, one concludes that in fact both sets $C\left(\omega_{1}, a\right), C\left(\omega_{2}, a\right) \subseteq \mathbb{R} \cup\{\infty\}$ are infinite. Indeed, suppose, say, that $\lim _{z \rightarrow a} \omega_{2}(z)=\omega_{2}(a)$. Then $C\left(\omega_{1}, a\right)$ must be infinite, so, as seen in the proof of Theorem 2.3, for any $c \in C\left(\omega_{1}, a\right)$, with at most three exceptions, there exists a sequence $\left\{z_{n}^{(c)}\right\}_{n \in \mathbb{N}}$ such that $\omega_{1}\left(z_{n}^{(c)}\right) \in c+\mathrm{i} \mathbb{R}$ and $\omega_{1}\left(z_{n}^{(c)}\right) \rightarrow c$ as $n \rightarrow \infty$. If $\omega_{2}(a) \in \mathbb{R}$, then

$$
\omega_{2}(a)=\lim _{n \rightarrow \infty} \frac{z_{n}^{(c)} \eta_{\mu}\left(\omega_{1}\left(z_{n}^{(c)}\right)\right)}{\omega_{1}\left(z_{n}^{(c)}\right)}=\frac{a}{c} \lim _{z \underset{\varangle}{ }{ }^{c}} \eta_{\mu}(z)=\eta_{\mu}(c) \frac{a}{c},
$$

for all $c \in C\left(\omega_{1}, a\right)$, with the possible exception of a subset of zero Lebesgue measure. Since the nontangential limit of $\eta_{\mu}$ exists at almost every point of $\mathbb{R}$, we can apply Theorem 1.5 to conclude that $\eta_{\mu}(z)=z \frac{\omega_{2}(a)}{a}$ for all $z \in \mathbb{C}^{+}$, and, by analytic continuation, for all $z \in \mathbb{C} \backslash[0,+\infty)$. This contradicts the fact that $\mu$ is not a point mass at $\omega_{2}(a) / a$. If $\omega_{2}(a)=\infty$, then we obtain the equality

$$
\frac{1}{c} \eta_{\mu}(c)=\lim _{n \rightarrow \infty} \frac{\eta_{\mu}\left(\omega_{1}\left(z_{n}^{(c)}\right)\right)}{\omega_{1}\left(z_{n}^{(c)}\right)}=\lim _{n \rightarrow \infty} \frac{\omega_{2}\left(z_{n}^{(c)}\right)}{z_{n}^{(c)}}=\infty
$$

for almost all $c \in C\left(\omega_{1}, a\right)$. This is impossible. Thus, both $C\left(\omega_{1}, a\right)$ and $C\left(\omega_{2}, a\right)$ must be infinite.

Suppose there exists a subset $E$ of $C\left(\omega_{1}, a\right)$ of nonzero Lebesgue measure such that $\eta_{\mu}(c) \in \mathbb{C}^{+}$for all $c \in E$. Then

$$
\eta_{\mu}(c)=\lim _{z \vec{\varangle}} \eta_{\mu}(z)=\lim _{n \rightarrow \infty} \eta_{\mu}\left(\omega_{1}\left(z_{n}^{(c)}\right)\right)=\lim _{n \rightarrow \infty} \eta_{\nu}\left(\omega_{2}\left(z_{n}^{(c)}\right)\right)=\lim _{n \rightarrow \infty} \eta_{\nu}\left(\frac{z_{n}^{(c)} \eta_{\mu}\left(\omega_{1}\left(z_{n}^{(c)}\right)\right)}{\omega_{1}\left(z_{n}^{(c)}\right)}\right)=\eta_{\nu}\left(\frac{a \eta_{\mu}(c)}{c}\right),
$$

which, by Theorem 1.5 , implies $\eta_{\mu}(z)=\eta_{\nu}\left(a \eta_{\mu}(z) / z\right)$ for all $z \in \mathbb{C}^{+}$, and hence for all $z \in \mathbb{C} \backslash[0,+\infty)$, which is a contradiction. 
If the nontangential limit of $\eta_{\mu}$ at $c$ is real for almost all $c \in C\left(\omega_{1}, a\right)$, and $\eta_{\mu}$ cannot be continued meromorphically through some point $x$ belonging to the interior of $C\left(\omega_{1}, a\right)$, we apply Proposition 1.9(b) to conclude that there exists a set $E \subset \operatorname{Int} C\left(\omega_{1}, a\right)$ of nonzero Lebesgue measure such that the set

$$
\left\{\lim _{z \rightarrow c} \eta_{\mu}(z) / z: c \in E\right\}
$$

is dense in $(-\infty, 0)$. Then

$$
\eta_{\mu}(c)=\lim _{z \vec{\varangle}} \eta_{\mu}(z)=\lim _{n \rightarrow \infty} \eta_{\mu}\left(\omega_{1}\left(z_{n}^{(c)}\right)\right)=\lim _{n \rightarrow \infty} \eta_{\nu}\left(\frac{z_{n}^{(c)} \eta_{\mu}\left(\omega_{1}\left(z_{n}^{(c)}\right)\right)}{\omega_{1}\left(z_{n}^{(c)}\right)}\right)=\eta_{\nu}\left(\frac{a \eta_{\mu}(c)}{c}\right),
$$

for all $c \in E$. By Privalov's theorem, $\eta_{\mu}(z)=\eta_{\nu}\left(\frac{a \eta_{\mu}(z)}{z}\right)$ for all $z \in \mathbb{C}^{+}$, and hence for all $z \in \mathbb{C} \backslash[0,+\infty)$, which is a contradiction.

If $\eta_{\mu}$ extends meromorphically through the interior of $C\left(\omega_{1}, a\right)$ and $\eta_{\nu}$ extends analytically through the interior of $C\left(\omega_{2}, a\right)$, then we obtain again that $\eta_{\mu}(z)=\eta_{\nu}\left(\frac{a \eta_{\mu}(z)}{z}\right)$ for all $z \in \mathbb{C} \backslash[0,+\infty)$, in the same way as in the proof of Theorem 2.3, which provides a contradiction.

Thus, the restriction of $\eta_{\mu \otimes_{\nu}}$ to the upper half-plane extends continuously to $\mathbb{R} \backslash\{0\}$. Since $\eta_{\mu \otimes_{v}}(\bar{z})=\overline{\eta_{\mu} \otimes_{v}(z)}$, $z \in \mathbb{C} \backslash[0,+\infty)$, we obtain that $\mathcal{D}$ is indeed empty. This concludes the proof.

Remark 3.3. The proof of Theorem 3.2 implies that whenever $\mu, v \in \mathcal{M}_{+}$, and neither of them is concentrated in one point, the restriction to the upper half-plane of the subordination functions $\omega_{1}$ and $\omega_{2}$ can be continuously extended to $\mathbb{R}$, including zero. Thus, a stronger result holds for the subordination functions than for $\psi_{\mu \bowtie \nu}$.

We have the following analogue of Corollary 2.5:

Corollary 3.4. If $\mu, v \in \mathcal{M}_{+}$, and none of them is concentrated at one point, then $\operatorname{supp}(\mu \otimes v)^{s}$ is closed and of zero Lebesgue measure. Moreover, $\operatorname{supp}(\mu \otimes v)^{\mathrm{sc}} \subset \operatorname{supp}(\mu \otimes v)^{\mathrm{ac}}$. In particular, $\mu \otimes v$ can never be singular.

Proof. As observed in Remark 1.11, $\psi_{\mu}(z)=\frac{1}{z} G_{\mu}\left(\frac{1}{z}\right)-1$ for all $z \in \mathbb{C} \backslash[0,+\infty)$. By Lemma 1.10, for $(\mu \otimes v)^{\mathrm{sc}_{-}}$ almost all $x$, the nontangential limit of $G_{\mu \nabla_{v}}$ at $x$, and thus of $\psi_{\mu \otimes_{v}}$ at $1 / x$, is infinite. According to Theorem 3.2, the restriction of $\psi_{\mu \bowtie v}$ to the upper half-plane extends continuously to $(0,+\infty)$, so the preimage of infinity under the extension of $\left.\left(\psi_{\mu \otimes_{v}}\right)\right|_{\mathbb{C}^{+}}$to $\mathbb{C}^{+} \cup(0,+\infty)$ (denoted also by $\left.\psi_{\mu \otimes_{v}}\right)$ is a closed set. Thus, the set $\left\{x \in \mathbb{R}: 1 / x \in \operatorname{supp}(\mu \otimes v)^{\mathrm{sc}}\right\}$ is included in the closed set $\psi_{\mu{ }_{\nu}}^{-1}(\{\infty\})$. According to Theorem 1.5, the Lebesgue measure of $\psi_{\mu \bigotimes \nu}^{-1}(\{\infty\})$ must be zero. Since, by Theorem 4.1 of [1], the number of atoms of $\mu \otimes v$ must be finite, we conclude that, indeed, $\operatorname{supp}(\mu \otimes v)^{\mathrm{s}}$ is a closed set of zero Lebesgue measure.

Let now $x \in \mathbb{R} \backslash\{0\}$ be such that $1 / x \in \operatorname{supp}(\mu \nabla v)^{\text {sc }}$. Since $\mu \otimes v$ can have only finitely many atoms, we may assume that $(\mu \otimes v)(\{1 / x\})=0$. Suppose that $1 / x \notin \operatorname{supp}(\mu \otimes v)^{\text {ac }}$. By the same argument as in the proof of Corollary 2.5, we obtain that $C\left(G_{\mu \bowtie v}, 1 / x\right)=-\mathbb{C}^{+} \cup \mathbb{R} \cup\{\infty\}$, so that $C\left(\psi_{\mu \otimes v}, x\right)=\mathbb{C}^{+} \cup \mathbb{R} \cup\{\infty\}$. This contradicts Theorem 3.2 .

\subsection{Measures supported on $\mathbb{T}$}

Fix $\mu, v \in \mathcal{M}_{*}$, and, as before, denote $\omega_{\mu, v}=\omega_{1}$, and $\omega_{v, \mu}=\omega_{2}$. According to Proposition 1.13(c), $\left|\eta_{\mu}(z)\right|<1$ for $z \in \mathbb{D}$, and $\eta_{\mu}(0)=0$. (Recall that $\mathbb{T}$ denotes the boundary of $\mathbb{D}$.)

Lemma 3.5. Let $\mu, v \in \mathcal{M}_{*}$, neither of them concentrated in one point. If there exists $a \in \mathbb{T}$ such that $\eta_{\mu}(z)=$ $\eta_{\nu}\left(a \eta_{\mu}(z) / z\right)$, then there exist $\alpha_{1}, \alpha_{2} \in \mathbb{T}$ and $t \in(0,1)$ such that $\mu=t \delta_{\alpha_{1}}+(1-t) \delta_{\alpha_{2}}$ and $v=t \delta_{\overline{a \alpha_{2}}}+(1-t) \delta_{\overline{a \alpha_{1}}}$.

Proof. The proof is similar to the proof of Lemma 3.1. Define $h_{\mu}(z)=a \eta_{\mu}(z) / z, h_{v}(z)=a \eta_{\nu}(z) / z, z \in \mathbb{D}$. The equation $\eta_{\mu}(z)=\eta_{\nu}\left(\frac{a}{z} \eta_{\mu}(z)\right)$ implies

$$
h_{\nu}\left(h_{\mu}(z)\right)=\frac{a}{h_{\mu}(z)} \eta_{\nu}\left(h_{\mu}(z)\right)=\frac{a}{h_{\mu}(z)} \eta_{\mu}(z)=z, \quad z \in \mathbb{D} .
$$


Applying $h_{\mu}$ to the previous inequality, we conclude as in the proof of Lemma 3.1 that $h_{\mu}$ and $h_{v}$ are conformal self-maps of the open unit disk $\mathbb{D}$, inverse to each other. Thus, there exist $b \in \mathbb{D}$ and $w \in \mathbb{T}$ such that

$$
h_{\mu}(z)=\frac{w z-b}{1-\bar{b} w z}, \quad \text { and } \quad h_{\nu}(z)=\bar{w} \frac{z+b}{1+\bar{b} z}, \quad z \in \mathbb{D} .
$$

But this implies that $\eta_{\mu}(z)=z h_{\mu}(z) / a=\left(w z^{2}-b z\right)(a-a \bar{b} w z)$, and $\eta_{\nu}(z)=\left(\bar{w}\left(z^{2}+b z\right)\right)(a+a \bar{b} z)$. So both $\eta_{\mu}$ and $\eta_{v}$ extend analytically through $\mathbb{T}$. This is possible only if $\mu$ and $v$ are purely atomic. According to [1], we have

$$
\mu(\{\alpha\})=\lim _{z \rightarrow \bar{\alpha}}(1-z \alpha) \psi_{\mu}(z),
$$

so, in our case, $\alpha$ is an atom if and only if

$$
\eta_{\mu}(\alpha)=\lim _{z \longrightarrow \bar{\alpha}} \eta_{\mu}(z)=1 .
$$

This shows that $\mu$ (and, by symmetry, $v$ ) must have exactly two atoms, namely the solutions of the equation $\eta_{\mu}(z)=1$ (and, respectively, $\eta_{\nu}(z)=1$ ). Let us focus on $\mu$. Suppose that $\mu=t \delta_{\alpha_{1}}+(1-t) \delta_{\alpha_{2}}, \alpha_{1}, \alpha_{2} \in \mathbb{T}, \alpha_{1} \neq \alpha_{2}$, so that

$$
\eta_{\mu}(z)=\frac{z\left(t \alpha_{1}+(1-t) \alpha_{2}\right)-z^{2} \alpha_{1} \alpha_{2}}{1-z\left((1-t) \alpha_{1}+t \alpha_{2}\right)} .
$$

We have seen before that $\eta_{\mu}(z)=\left(z^{2} w-z b\right)(a-a \bar{b} w z)$. Evaluating $\eta_{\mu}(z) / z$ in $z=0$ and $z=1$ will give

$$
t \alpha_{1}+(1-t) \alpha_{2}=-b / a \text { and } \alpha_{1} \alpha_{2}=-w / a .
$$

A straightforward calculation will give

$$
\eta_{\nu}(z)=\frac{z\left(t \overline{a \alpha_{2}}+(1-t) \overline{a \alpha_{1}}\right)-z^{2} \overline{a \alpha_{2}} \overline{a \alpha_{1}}}{1-z\left((1-t) \overline{a \alpha_{2}}+t \overline{a \alpha_{1}}\right)},
$$

so $v=t \delta_{\overline{a \alpha_{2}}}+(1-t) \delta_{\overline{a \alpha_{1}}}$. This concludes the proof.

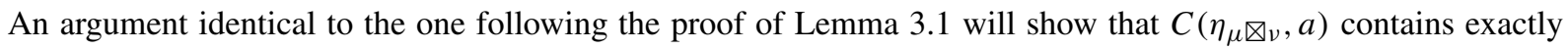
one point for any $a \in \mathbb{T}$ whenever $\mu$ and $v$ are as in Lemma 3.5. We have the following

Theorem 3.6. Let $\mu, v \in \mathcal{M}_{*}$, none of them concentrated in one point. Then the set

$$
\mathcal{E}_{1}=\left\{a \in \mathbb{T}: C\left(\eta_{\mu \otimes_{v}}, a\right) \cap \mathbb{D} \text { is infinite }\right\}
$$

is empty. If $\operatorname{supp} \mu \neq \mathbb{T}$ and $\operatorname{supp} v \neq \mathbb{T}$, then the set

$$
\mathcal{E}=\left\{a \in \mathbb{T}: C\left(\eta_{\mu \nabla_{\nu}}, a\right) \text { is infinite }\right\}
$$

is empty.

Let us note that $\mathcal{E}$ is empty if and only if $\lim _{z \rightarrow a} \eta_{\mu \otimes_{\nu}}(z)$ exists for all $a \in \mathbb{T}$, or equivalently, $\eta_{\mu{ }_{\nu}}$ extends continuously to $\overline{\mathbb{D}}$.

Proof. Using the fact that $\eta_{\mu}(\mathbb{D}) \subseteq \mathbb{D}$, provided by Proposition 1.13(c), and Proposition 1.9(a), the proof becomes a straightforward adaptation of the proofs of Theorems 2.3 and 3.2. We omit the details.

Corollary 3.7. If $\operatorname{supp} \mu \neq \mathbb{T}$, $\operatorname{supp} v \neq \mathbb{T}$, then $\operatorname{supp}(\mu \otimes v)^{\mathrm{s}}$ a closed set of zero linear measure. Moreover, $\operatorname{supp}(\mu \otimes v)^{\mathrm{sc}} \subset \operatorname{supp}(\mu \otimes v)^{\mathrm{ac}}$. In particular, $\mu \otimes v$ can never be singular (the last statement holds for arbitrary elements in $\left.\mathcal{M}_{*}\right)$.

Proof. Let us start by recalling that, as observed in Remark 1.12, the real part of the function $\frac{1}{\pi}\left(\psi_{\mu \nabla_{v}}(z)+\frac{1}{2}\right)$ is the Poisson integral of the measure $d(\mu \nabla v)(\bar{t})$. Thus, as seen in Lemma 1.10, for any $x \in \mathbb{T}$ outside a set of $(\mu \nabla v)^{\mathrm{sc}_{-}}$ measure zero, the real part of $\psi_{\mu \otimes_{v}}$ will converge to infinity as $z$ approaches $\bar{x}$ nontangentially. (We recall that the 
imaginary part of the Cauchy transform of a probability measure on $\mathbb{R}$ is, up to a constant, the Poisson integral of the measure.)

Assume first that $\operatorname{supp} \mu \neq \mathbb{T}$, and $\operatorname{supp} v \neq \mathbb{T}$. For $(\mu \otimes v)^{\mathrm{sc}}$-almost all $x \in \mathbb{T}$, the nontangential limit of $\psi_{(\mu \otimes v)^{\mathrm{s}}}$ (respectively of $\eta_{(\mu \bigotimes \nu)^{\mathrm{s}}}$ ) at $\bar{x}$ is infinity (respectively 1). Since, by Theorem 3.6, $\eta_{\mu \otimes_{\nu}}$ extends continuously to $\overline{\mathbb{D}}$, the preimage of 1 via $\eta_{\mu \bigotimes v}$ is a closed set. According to Privalov's theorem (Theorem 1.5), the preimage of 1 via the extension of $\eta_{\mu \otimes_{v}}$ to $\overline{\mathbb{D}}$ must be of zero linear measure. On the other hand, as shown in [1], Theorem 3.1, $\mu \otimes v$ can have only finitely many atoms. Thus, the support of $(\mu \nabla v)^{\mathrm{s}}$ is a closed set of zero linear measure in $\mathbb{T}$.

Let now $x \in \mathbb{T}$ be such that $\bar{x} \in \operatorname{supp}(\mu \nabla v)^{\mathrm{sc}}$. Since $\mu \nabla v$ has only finitely many atoms, there is no loss of generality in assuming that $\bar{x}$ is not an atom of $\mu \otimes v$. Assume to the contrary that $\bar{x}$ does not belong to $\operatorname{supp}(\mu \otimes v)^{\mathrm{ac}}$. Then there exists a neighborhood $V$ of $x$ in $\mathbb{T}$ such that the Poisson integral of $d(\mu \otimes v)(\bar{t})$ has nontangential limit equal to zero at all points of $V$, outside a set of linear measure zero. But $\{\bar{a}: a \in V\} \cap \operatorname{supp}(\mu \nabla \nu)^{\mathrm{sc}}$ is an infinite set. Since $\varangle \lim _{z \rightarrow \bar{a}} \frac{1}{\pi}\left(\psi_{\mu \bigotimes v}(z)+\frac{1}{2}\right)=\infty$ for $(\mu \otimes v)^{\mathrm{sc}}$-almost all $a \in \operatorname{supp}(\mu \nabla v)^{\mathrm{sc}}$ and $x \in V, \bar{x} \in \operatorname{supp}(\mu \otimes \nu)^{\mathrm{sc}}$, we

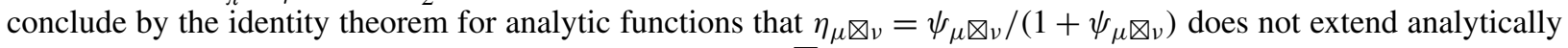
through $x$. Thus, according to Theorem 1.7, $C\left(\eta_{\mu \otimes_{v}}, x\right)=\overline{\mathbb{D}}$. This contradicts Theorem 3.6.

Suppose now that $\mu, v$ have arbitrary supports in $\mathbb{T}$, and assume that $\mu \otimes v$ is purely singular. This will imply that the nontangential limit of $\eta_{\mu \bowtie v}$ will have absolute value one at all points $x \in \mathbb{T}$, with the possible exception of a set of linear measure zero. Since $\mu \otimes v$ cannot be purely atomic, it must have a singular continuous part. Let $\bar{x} \in \operatorname{supp}(\mu \otimes \nu)^{\mathrm{sc}}$. As we have seen above, $\eta_{\mu \bigotimes v}$ does not extend analytically through points in $\operatorname{supp}(\mu \otimes \nu)^{\mathrm{sc}}$. Thus, according to Theorem 1.7, $C\left(\eta_{\mu{ }_{v}}, x\right)=\overline{\mathbb{D}}$. This contradicts Theorem 3.6.

\section{Acknowledgements}

I am grateful to the referees for numerous useful suggestions and for pointing out some inaccuracies in the proofs.

\section{References}

[1] S.T. Belinschi, The atoms of the free multiplicative convolution of two probability distributions, Integral Equations Operator Theory 46 (4) (2003) 377-386.

[2] S.T. Belinschi, H. Bercovici, Atoms and regularity for measures in a partially defined free convolution semigroup, Math. Z. 248 (4) (2004) 665-674.

[3] S.T. Belinschi, H. Bercovici, Partially defined semigroups relative to multiplicative free convolution, Int. Math. Res. Not. 2 (2005) 65-101.

[4] H. Bercovici, Personal communication.

[5] H. Bercovici, D. Voiculescu, Convolutions of measures with unbounded support, Indiana Univ. Math. J. 42 (3) (1993) $733-773$.

[6] H. Bercovici, D. Voiculescu, Regularity questions for free convolution, in: Nonselfadjoint Operator Algebras, Operator Theory, and Related Topics, in: Oper. Theory Adv. Appl., vol. 104, Birkhäuser, Basel, 1998, pp. 37-47.

[7] P. Biane, On the free convolution with a semi-circular distribution, Indiana Univ. Math. J. 46 (3) (1997) 705-718.

[8] P. Biane, Processes with free increments, Math. Z. 227 (1) (1998) 143-174.

[9] P. Billingsley, Probability and Measure, Wiley Ser. Probab. Math. Statist., Wiley, New York, 1995.

[10] E.F. Collingwood, A.J. Lohwater, The Theory of Cluster Sets, Cambridge University Press, Cambridge, 1966.

[11] P.L. Duren, Theory of $H^{p}$ Spaces, Academic Press, New York, 1970.

[12] S. Saks, Theory of the Integral, Monografie Matematyczne, Warszawa, 1937.

[13] E. Stein, G. Weiss, Introduction to Fourier Analysis on Euclidean Spaces, Princeton Math. Ser., vol. 32, Princeton University Press, Princeton, NJ, 1971

[14] D. Voiculescu, Multiplication of certain noncommuting random variables, J. Operator Theory 18 (2) (1987) 223-235.

[15] D. Voiculescu, The analogues of entropy and of Fisher's information measure in free probability theory. I, Comm. Math. Phys. 155 (1) (1993) 411-440.

[16] D.V. Voiculescu, K.J. Dykema, A. Nica, Free Random Variables, CRM Monogr. Ser., vol. 1, American Mathematical Society, Providence, RI, 1992. 